\title{
Ultraviolet Photo Catalytic Oxidation (UVPCO) Sensor for Air and Surface Sanitizers Using CS Amplifier
}

\author{
Pawan Whig ${ }^{1, *}$ and Syed Naseem Ahmad ${ }^{2}$ \\ ${ }^{1}$ Vivekananda Institute of Professional Studies, Delhi 110034, India \\ ${ }^{2}$ Department of ECE, Jamia Millia Islamia, New Delhi 110025, India; snahmad@jmi.ac.in \\ * Correspondence: pawanwhig@gmail.com
}

\begin{abstract}
In this research paper a novel Ultra Violet Photo Catalyst Oxidation (UVPCO) sensor for air and surface sanitization using Common Source (CS) amplifier is presented. The ultra violet photo catalysis is the process in which the highly reactive radicals like $\mathrm{H}^{+}, \mathrm{OH}$-and peroxides ions are produced from air in the presence of the ultra violet radiation and photo catalyst. In this process, the free radicals outbreaks the bio aerosols like bacteria, fungus and volatile organic compounds (VOCs) and destroy them. The proposed system is relies on the fast operation of PCS which operates under sub-threshold conditions and reduced computation time. The properties of common source amplifier like very high voltage gain and input output resistance increased the sensitivity as well as stability of the circuit. The system is more user friendly and the outcomes of simulation are fairly in agreement with the theoretical estimation.
\end{abstract}

Keywords: bio aerosol; Photo Catalytic Oxidation (PCO); hydroxyl; hydrogen peroxide; SPICE; surface sanitizer

\subsection{Introduction}

Now day's air pollution is increasing because of harmful material in earth atmosphere causing infections, diseases, deaths, and damage to crop. These harmful material can be man-made or natural origin. The main source of these harmful material are power plant and industrialised factories. The main air pollutant which can have adversarial effects on humans and the ecosystem are sulphur oxide (SOx), nitrogen oxide $(\mathrm{NOx})$, carbon monoxide $(\mathrm{CO})$, volatile organic compound, chlorofluorocarbons (CFCs), ammonia $\left(\mathrm{NH}_{3}\right)$ and some toxic metals. These air pollutants can have adverse effect on human health like headache, fatigue, poor memory, respiratory irritation, pneumonia in children, lung cancer, heart disease, eye and throat irritation. Hence, there is a need to purify air by using advanced process called Photo Catalytic Oxidation (PCO).

In PCO, Titanium dioxide used as a catalyst which cleans the air [1]. As air falls on this catalyst, electron will get energy and released at its surface. Then it will interact 
with water molecules in the air, as we know that electron having energy which break the water molecules into hydroxy1 radicals, hydroxide and hydrogen peroxide.

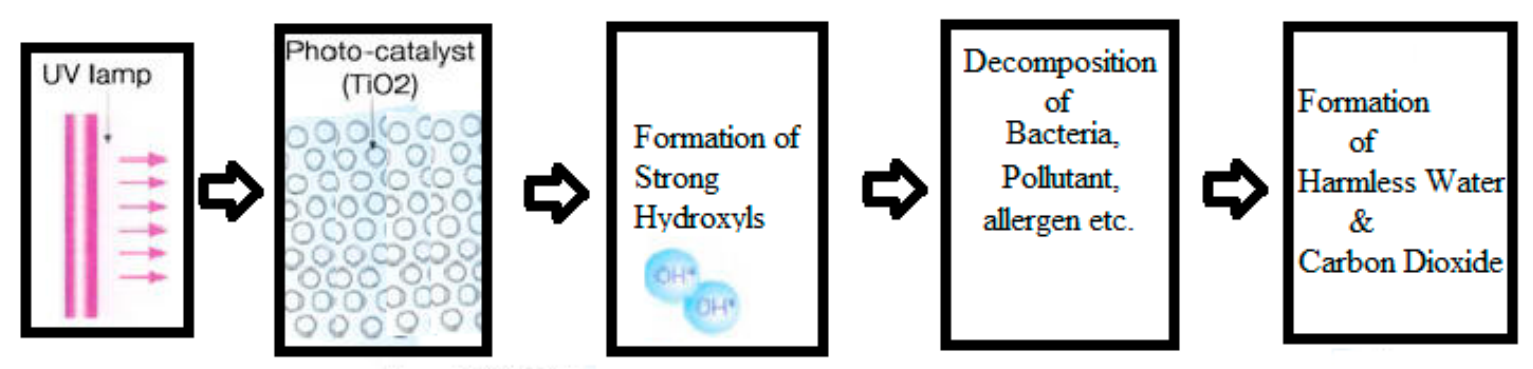

Fig.1 Process of Photo Catalytic Oxidation

Being reactive these hydroxy1 radicals attack organic pollutant molecules, breaking them into carbon dioxide and water which are harmless substance and thus help in cleaning environment. The conventional method used for $\mathrm{TiO}_{2} \mathrm{PCO}$ applications includes bulky and complex set ups and require ample time for computation. The complete photo catalytic oxidation process is shown in Figure 1 This process is responsible for purify air up to significant level, to measure that level there is a serious need of sensor which tell the percentage purification of air.

Semiconductor technology is very popular and widely used for sensor development as it provides an advantage of low power, high speed, small size integration and their signal processing capability. More often, Computer aided design tools are used which provide simulation and synthesis of semiconductor sensors [2]. Spice has built in models for most semiconductor devices but there is scarcity of appropriate models available for semiconductor sensors. This approach requires a deep knowledge of the code structure, subroutines and it is strictly linked to a particular version of Spice [3]. In this paper, spice macro model for UVPCO is used in Tanner tool version 15 for 70nm technology. This tool contains a large set of MOSFET models which operate in sub threshold region. The simplest PCS is $\mathrm{O}_{2}$ sensitive where the sensitive surface is made up of insulator layer like Titanium Oxide $\left(\mathrm{TiO}_{2}\right)$ exposed to an electrolyte solution. A p-type semiconductor and $\mathrm{TiO}_{2}$ insulator are placed into aqueous electrolyte solution where the response of PCS to $\mathrm{O}_{2}$ can be explained in terms of photo catalysis. In the submicron level the thickness of gate oxide is very small in dimension. As the dimension of gate oxide decreases this results in tunneling of charge carriers which significantly increases the leakage current of MOSFETS and results in the decrease of device reliability.

One way to continue scaling is possible by using materials having much higher permittivity than silicon dioxide. 


$$
\mathrm{Eg}_{\mathrm{g}} \approx 20\left[\frac{3}{2+\epsilon}\right]^{2}
$$

Where $\mathrm{Eg}_{\mathrm{g}}$ is band gap $\in$ permittivity of the dielectric.

The relationship is given in eq. 1 between the band gap and the permittivity. The inverse relation of permittivity of dielectric shows that the band gap decreases with increase in the value of permittivity. The band gap of titanium oxide is above $3.5 \mathrm{eV}$ which restrict tunneling. The capacitance in accumulation is generally found to depend on gate bias. But as compared to Capacitance Voltage (CV) curve of $\mathrm{SiO}_{2}$ capacitor, this effect is less pronounced in $\mathrm{TiO}_{2}$ capacitor sensor [7-8].

The magnitude of the interface state density for $\mathrm{TiO}_{2}$ capacitor is given in equation (2).

$$
\mathrm{DIT}=\left[\frac{G_{p}}{w}\right]_{w_{p}}\left[f_{D}\left(\sigma_{s}\right)\right]^{-1}
$$

Where $f_{D}$ is a peak conductance frequency given as

$\mathrm{fD}\left(\sigma_{s}\right)=\frac{1}{2 \xi_{p} \sqrt{2 \pi \sigma_{s}}} \int_{-\infty}^{+\infty} e^{-\frac{\eta^{2}}{2 \sigma_{s}^{2}}} e^{-\eta} \ln \left[1+\xi_{p}^{2} e^{2 \eta}\right] d \eta$

The width of accumulation layer is determined by the wave function of the Eigen states. The solution of the Schrodinger equation at the interface leads to Airy function which produces an average position for accumulation of carriers in the ith energy state which is given in eq. 4

$$
E i \approx\left[\frac{h^{2}}{8 \pi^{2} m^{*}}\right]^{\frac{1}{3}}\left[\frac{3 \pi}{2} q F_{S}\left(i+\frac{3}{4}\right)\right]^{\frac{2}{3}}
$$

Where $\mathrm{q}$ is the electron charge, $\mathrm{F}_{\mathrm{s}}$ is the electric field at the surface, $\mathrm{E}_{\mathrm{i}}$ is the ith energy level, $\mathrm{m}^{*}$ is the effective mass and $\mathrm{h}$ is Plank's constant. For reasonable assumptions the accumulation layer thickness (x) should be of the order of $40 \AA$ [9-11]. Thus the effect is small for $\mathrm{SiO}_{2}$ layers with thickness much greater than $15 \AA$, while the effect is quite noticeable in much thicker $\mathrm{TiO}_{2}$ films. Taking this effect into account a series capacitance is added to $\mathrm{C}_{\mathrm{ox}}$ which is called quantum capacitance $\mathrm{C}_{\mathrm{q}}$.

$$
\frac{1}{C_{M}}=\frac{1}{C_{O X}}+\frac{1}{C_{q}}=\frac{t_{T^{2} o_{2}}}{A \varepsilon_{T i o_{2} \varepsilon_{O}}}+\frac{\langle x\rangle}{A \varepsilon_{S i} \varepsilon_{O}}
$$

The main reason of employing a CMOS Based common source PCS circuitry, is the fact that the fluctuation of ions influences the threshold voltage, which is internal parameter of the PCS and can manifest itself as a voltage signal at output but as a function of the trans conductance. The trans conductance is a passive parameter and in order to derive voltage or current signal from its fluctuations the sensor has to be attached to conditioning and transmitting circuit. 


\subsection{Common Source Amplifier}

In the case of CS amplifier, source of the input transistor is connected to ground. The source is the reference point for both the input signal and for the output voltage. Gate of the input transistor is the input node; its drain is output node as shown in Figure 2. There is a resistance $\mathrm{R}$ between the output node and the positive supply VDD. The resistance can be realized as resistor or as transistor acting as current source. The small signal model of common source amplifier is shown in Figure 3.

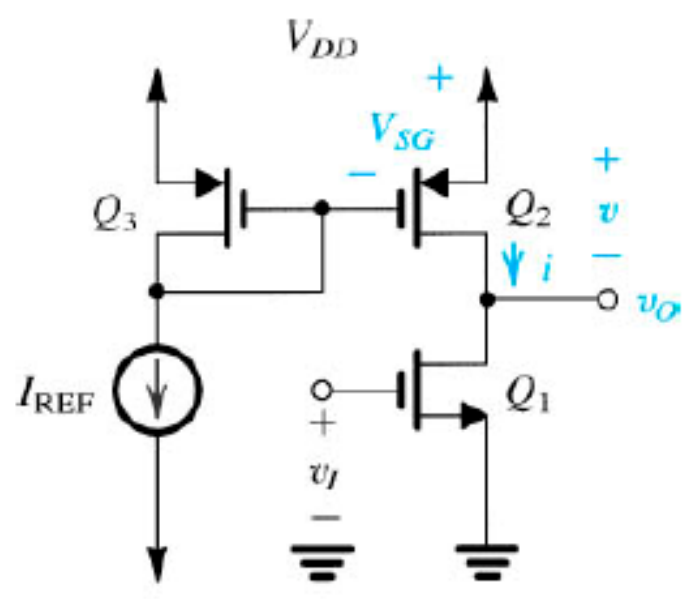

Fig. 2 Basic circuit of common source amplifier

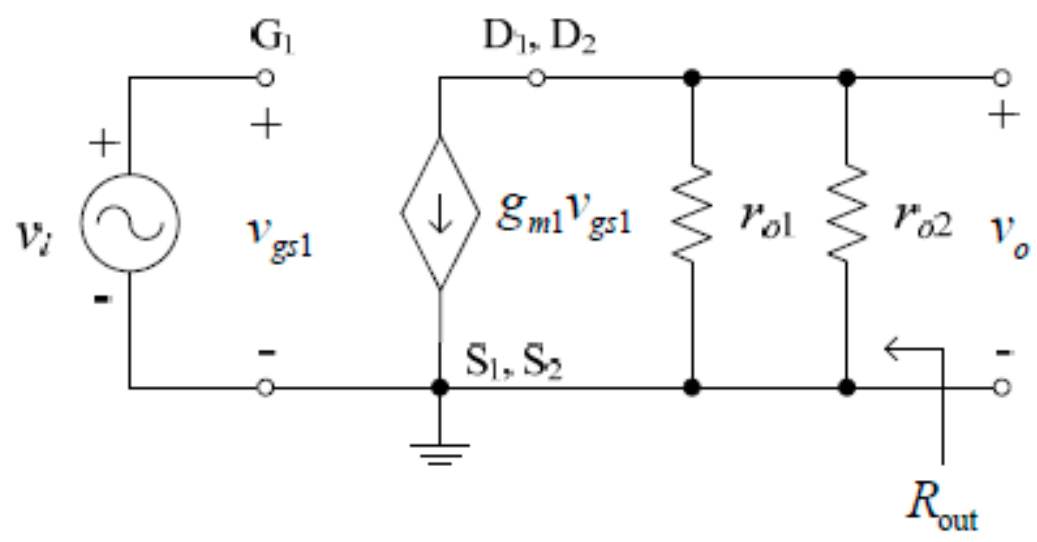

Fig.3 Small signal model of common source amplifier

$A_{v o}=-\frac{\sqrt{2 K_{n} \frac{W}{L}}}{\frac{1}{V_{A 1}}+\frac{1}{V_{a 2}} \sqrt{I_{R E F}}}$

$R_{\text {out }}=r_{\text {o1 }} \| r_{02}$ 
The small signal model of common source amplifier have high voltage gain, and input output resistance which increased the sensitivity of the circuit.

\subsection{PCS Macro model}

The PCS is just like a MOSFET the only difference in their construction is that the gate terminal is connected with chip in the form of reference electrode placed in an aqueous solution. The current - voltage (CV) relationship in non-saturated mode for PCS is same as that of MOSFET as given below in eq. 8.

$\mathrm{I}_{\mathrm{d}}=\mathrm{Cox}_{\mathrm{ox}} \frac{\mathrm{W}}{\mathrm{L}}\left[\left(\mathrm{V}_{\mathrm{gs}}-\mathrm{V}_{\mathrm{t}}\right) \mathrm{V}_{\mathrm{ds}}-\frac{1}{2} \mathrm{~V}_{\mathrm{ds}}{ }^{2}\right]$

Where Cox is the oxide capacity per unit area, $\mu$ is the electron mobility in the channel, $\mathrm{W}$ and $\mathrm{L}$ the width and the length of the channel, respectively. In the above relation $I_{d}$ is made sole function of $V_{g s}$ only if all other parameters like $\beta=\mu \operatorname{Cox} W / L, V_{d s}, V_{t}$ are taken constant. The relationship of threshold voltage with other parameters is given below in eq. 9 .

$$
\mathrm{V}_{\mathrm{t}}=\frac{\phi_{\mathrm{M}}-\phi_{\mathrm{si}}}{\mathrm{q}}-\frac{\mathrm{Q}_{\mathrm{ox}}+\mathrm{Q}_{\mathrm{sS}}+\mathrm{Q}_{\mathrm{B}}}{\mathrm{C}_{\mathrm{ox}}}+2 \phi_{\mathrm{f}}
$$

Where

$\phi_{\mathrm{M}}:$ work function of gate metal.

$\phi_{\mathrm{si}}$ : work function of silicon gate.

Qox: oxide charge.

$\mathrm{Q}_{\mathrm{ss}}$ : Interface charge carriers.

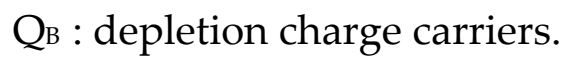

The fabrication steps follows in PCS is same as MOSFET, as a result of which the threshold voltage equation of PCS resembles with MOSFET. Although the concept of forming the equation is same but there are two additional terms added.

(1) Constant reference electrode potential Eref.

(2) Interfacial potential which consists of chemical input parameter $(\Psi)$ which depends upon concentration of $\mathrm{O}_{2}$ in the solution and surface dipole potential $\left(\chi^{\text {sol }}\right)$.

The above mentioned two parameters along with eq. 9 constitute the resultant threshold eqn. of the PCS shown below in eq. 10.

$\mathrm{V}_{\text {th }(\mathrm{PCS})}=\mathrm{ERef}_{\mathrm{Ref}}-\Psi_{\mathrm{sol}}+\chi^{\mathrm{sol}}+\frac{-\phi_{\mathrm{s}}}{\mathrm{q}}-\frac{\mathrm{Q}_{\mathrm{ox}}+\mathrm{Q}_{\mathrm{ss}}+\mathrm{Q}_{\mathrm{B}}}{\mathrm{C}_{\mathrm{ox}}}+2 \phi_{\mathrm{f}}$

According to the characteristics of the MOSFET gate to source voltage, Vgs known as reference voltage drain current is allowed to vary with drain to source voltage 
keeping reference voltage constant. Comparing PCS with MOSFET keeping the concentration of $\mathrm{O}_{2}=1 \mathrm{mg} / \mathrm{it}$ is found that the curve resembles with the characteristic Vds /Ids curve of MOSFET keeping Vgs constant as shown in Fig.4. Now keeping the reference voltage $\mathrm{Vgs}=0$ it is observed that for different concentration levels of $\mathrm{O}_{2}$, different $\mathrm{Vds} / \mathrm{Ids}$ curves are obtained as shown in Fig.5. From the above it is observed that as the oxygen concentration level decreases saturation cut off current $\mathrm{I}_{\mathrm{d} s}$ increases hence it is concluded that PCS can be treated as MOSFET on the basis that the chemical input parameter $\Psi_{\text {sol }}$ is a function of $\mathrm{O}_{2}\left(\Psi_{\text {sol }}=\mathrm{f}(\mathrm{Oxygen})\right)$.

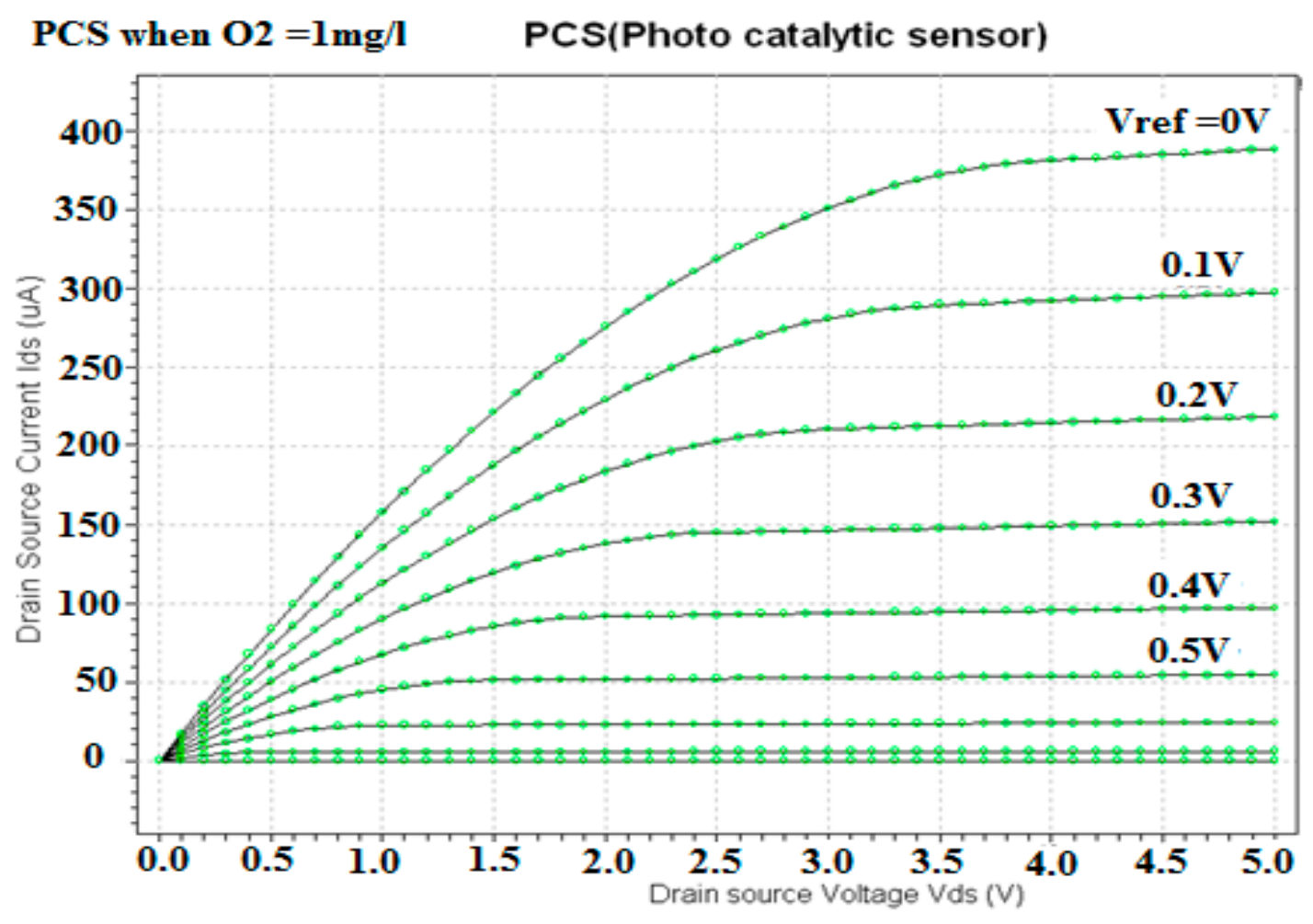

Fig. 4. Id/Vds curves can be recorded as function of Vgs 


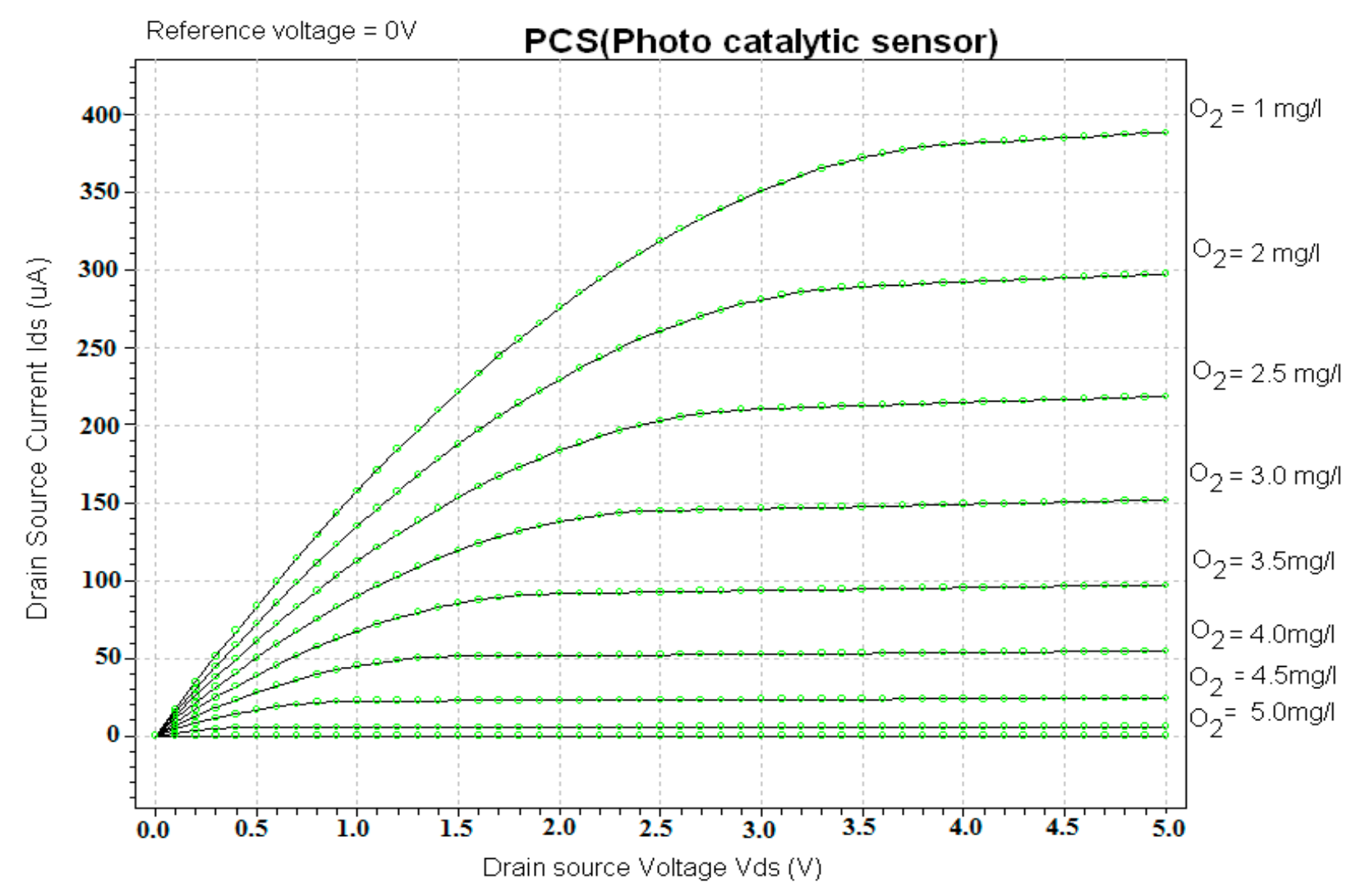

Fig.5. Id/Vds curves when the reference electrode connected to the source $(\operatorname{Vgs}=0)$

\subsection{Device description and Analysis}

The PCS generates potential proportional to activity of detected oxygen ion. Potential in PCS is measured against the reference electrode. The common source configuration has been used to measure the change in the concentration of dissolved oxygen through a corresponding shift in the device threshold voltage. The sensing readout circuit detect the ion concentration of the solution with the feature of CVCC operation mode and floating reference electrode which made the design simple and robust. In this configuration, two constant voltage sources $0.7 \mathrm{~V}$ and $0.2 \mathrm{~V}$ are fed to the two positive terminals of the amplifiers causes the drain terminal and the source terminal of the PCS to keep a constant drain source voltage difference of 0.5V.To maintain and operate the PCS in the linear region, the gate to source voltage variation of PCS threshold voltage should be directly proportional to the variations of the dissolved oxygen values. Potential difference between the gate sensing membrane and the reference electrode is determine by the ion concentration of the solution. The readout circuit is to be implemented by integrated circuit. The measured signal is the output from amplifier 


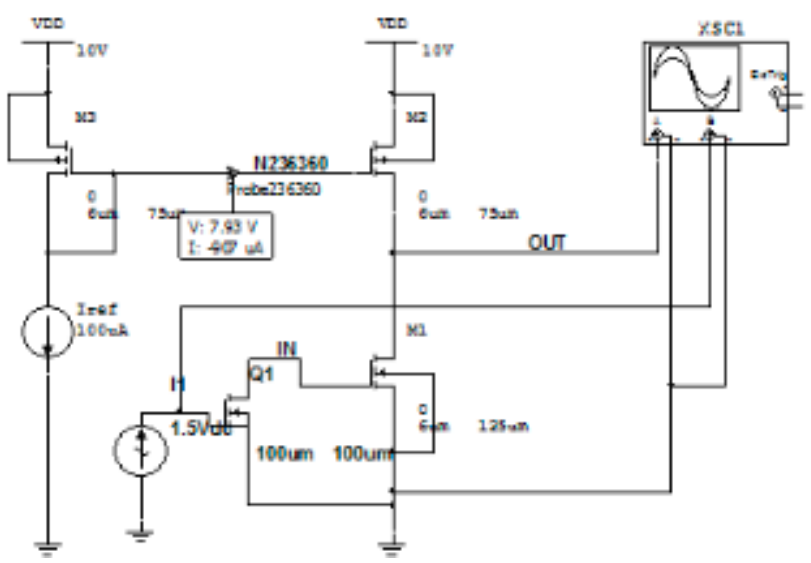

Fig. 6. Simulated Circuit diagram of PCS

\section{Fourier analysis}

Fourier analysis is a method of analysing complex periodic waveforms. It permits any non-sinusoidal period function to be resolved into sine or cosine waves and a DC component. This permits further analysis and allows you to determine the effect of combining the waveform with other signals. Each frequency component of the response is produced by the corresponding harmonic of the periodic waveform. Each term is considered a separate source. According to the principle of superposition, the total response is the sum of the responses produced by each term. It is observed that, amplitude of the harmonics decreases progressively as the order of the harmonics increases. This indicates that comparatively few terms yield a good approximation. Fourier analysis of the device is shown in Figure 7.

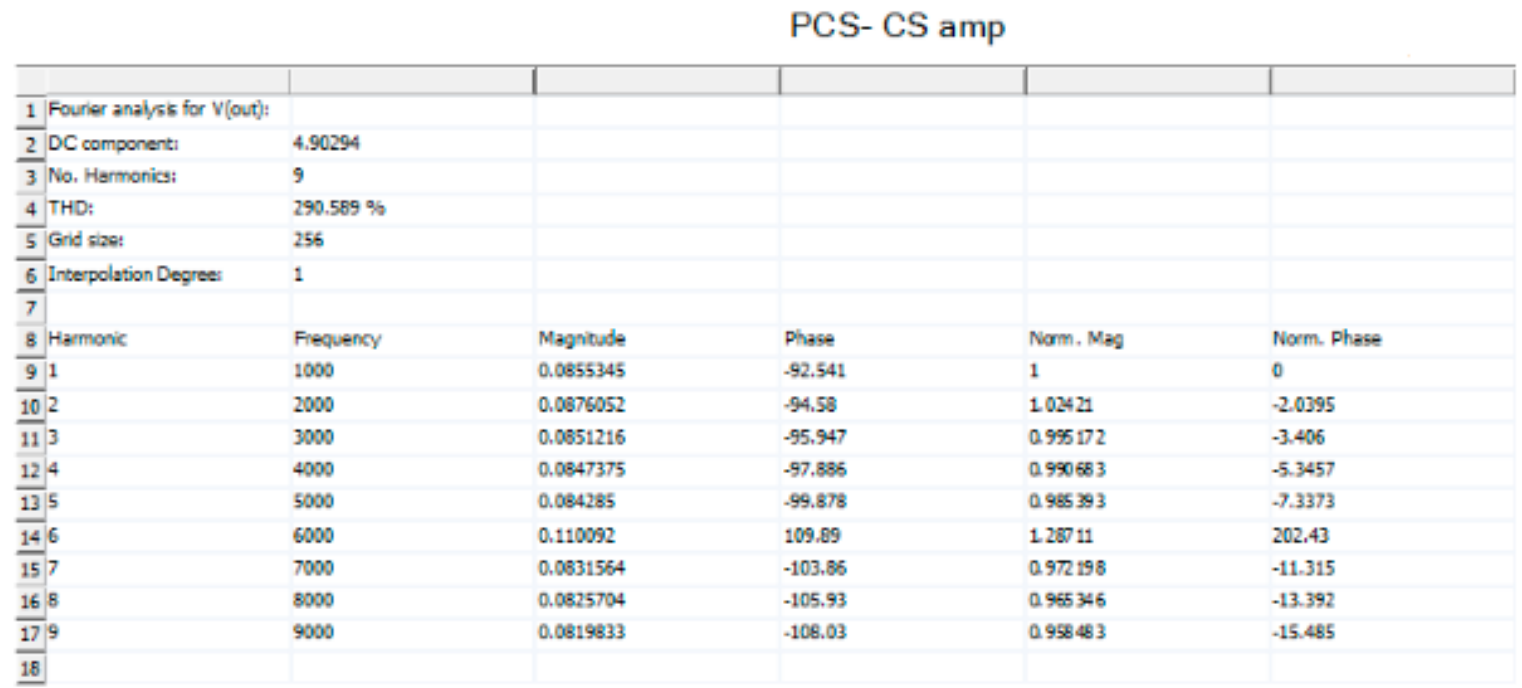

Fig. 7. Fourier analysis of the device 


\section{Monte Carlo analysis}

Monte Carlo Analysis is a statistical technique that explore how changing component properties affects circuit performance. Monte Carlo Analysis will perform DC, AC or Transient Analysis and vary the component properties. The Triangular outcome defines the minimum, most likely, and maximum values. The Monte Carlo analysis of the device is shown in figure 8. Values around the most likely are more likely to occur. Variables that could be described by a triangular distribution include past information about signal per unit of time. In Monte Carlo simulation, it's easy to see which inputs had the biggest effect on bottom-line results.

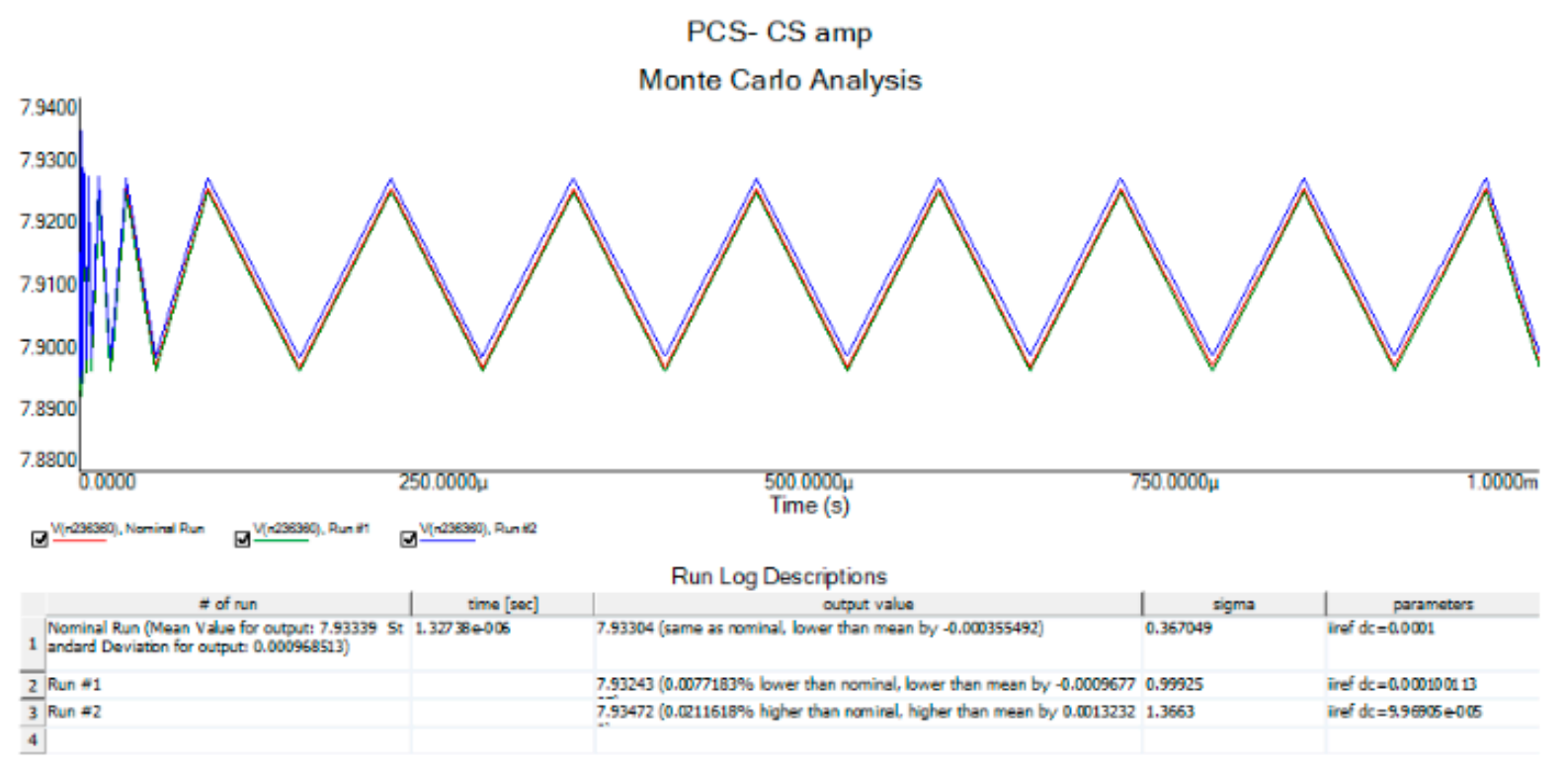

Fig. 8. Monte Carlo analysis of the device

\section{Temperature Sweep}

Using Temperature Sweep Analysis, one can quickly verify the operation of circuit by simulating it at different temperatures. The effect is the same as simulating the circuit several times, once for each temperature. Figure 9 shows the variation of output with temperature for a given device. It is found that as the temperature increases, the output of the device decreases. 


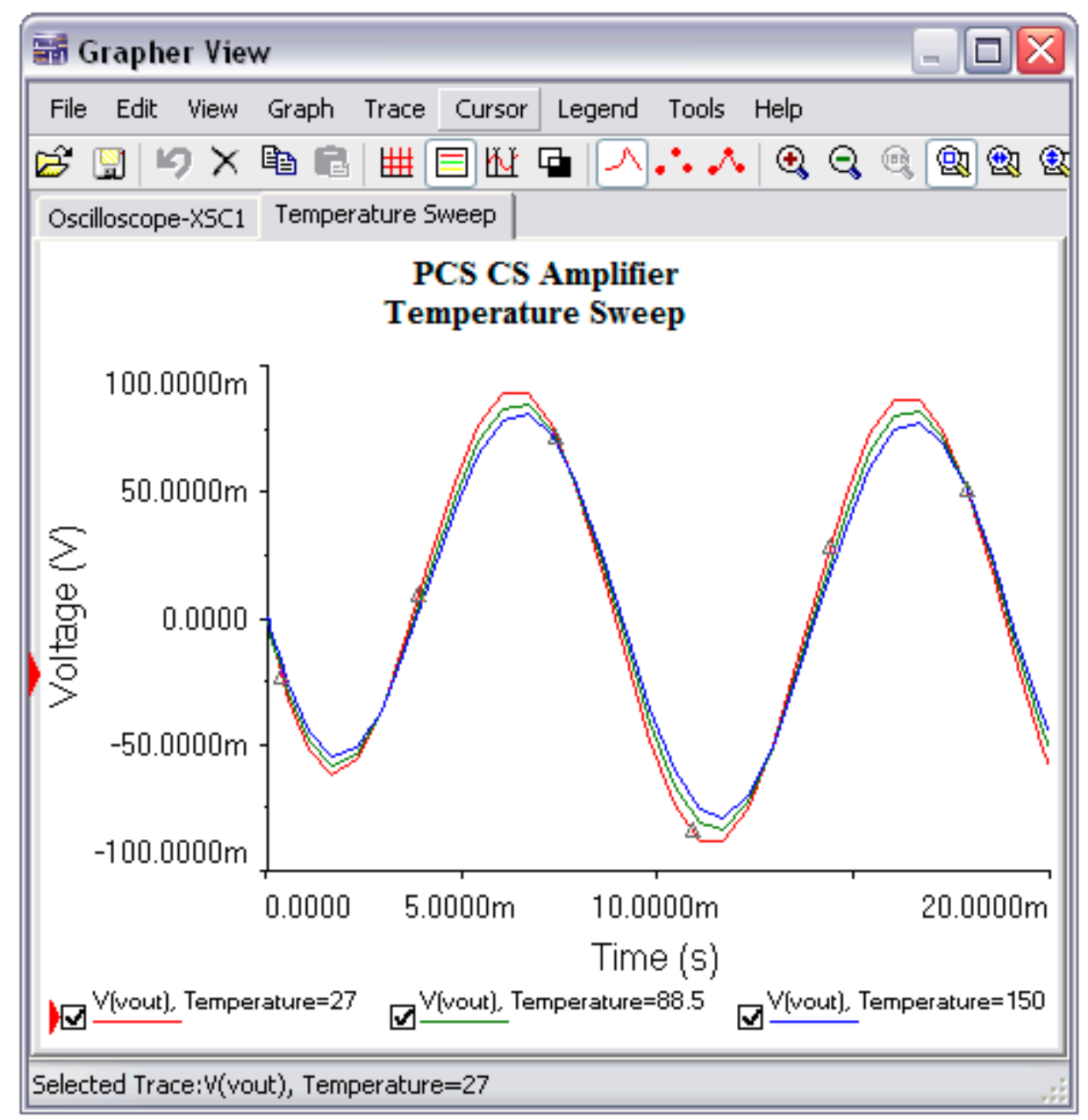

Fig. 9. Temperature sweep analysis of the device

\section{AC Analysis}

AC Analysis is used to calculate the frequency response of linear circuits. In AC Analysis, the DC operating point is first calculated to obtain linear, small-signal models for all nonlinear components. Then a complex matrix (containing both real and imaginary component parts) is created. To construct a matrix, DC sources are given zero values. AC sources, capacitors, and inductors are represented by their AC models. Nonlinear components are represented by linear AC small-signal models, derived from the DC operating point solution. All input sources are considered to be sinusoidal. The frequency of the sources is ignored. If the function generator is set to a square or triangular waveform, it will automatically switch internally to a sinusoidal waveform for analysis. AC Analysis then calculates the AC circuit response as a function of frequency. AC analysis of the given device is shown in Fig. 10. 


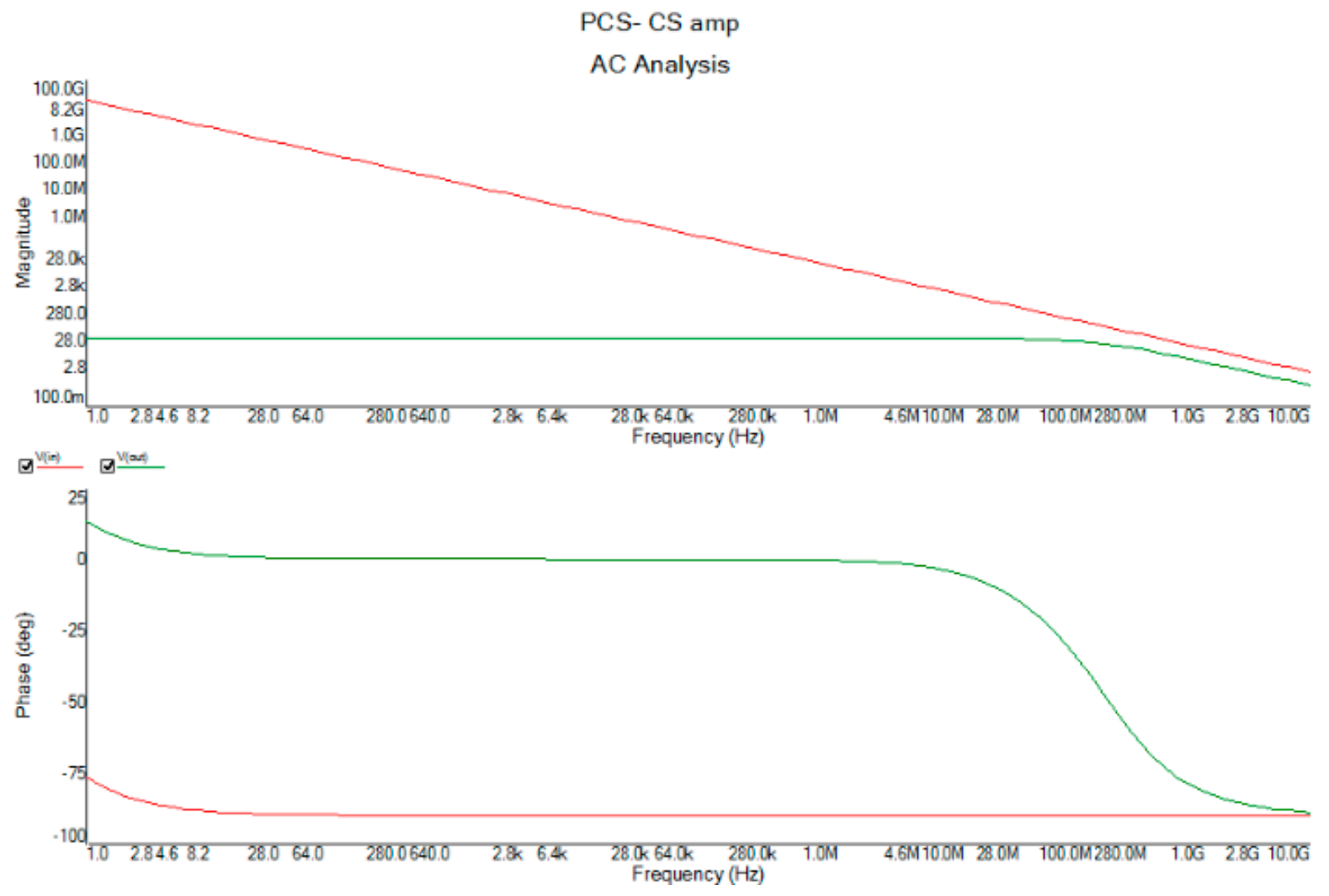

Fig. 10. AC analysis of the device

The Regression statistics including multiple R, R square Adjusted R square and Standard error obtained during experiment is shown in Table 1.

Table 1 Regression Statistics

\begin{tabular}{|c|c|}
\hline \multicolumn{2}{|c|}{ Regression Statistics } \\
\hline Multiple R & 0.983 \\
\hline R Square & 0.966 \\
\hline Adjusted R Square & 0.960 \\
\hline Standard Error & 0.026 \\
\hline Observations & 8.000 \\
\hline
\end{tabular}


Table 2 Variation of Oxygen concentration with maximum current decrease

\begin{tabular}{|c|c|}
\hline $\begin{array}{c}\text { Oxygen } \\
\text { Concentration }\end{array}$ & $\begin{array}{c}\text { Maximum Current } \\
\text { decrease }\end{array}$ \\
\hline 0 & 0 \\
\hline 0.5 & 0.08 \\
\hline 1 & 0.16 \\
\hline 1.5 & 0.21 \\
\hline 2 & 0.216 \\
\hline 2.5 & 0.32 \\
\hline 3 & 0.35 \\
\hline 3.5 & 0.37 \\
\hline
\end{tabular}

\section{Normal Probability Plot}

The normal probability plot is a special case of the probability plot. The points on this plot form a nearly linear pattern, which indicates that the normal distribution is a good model for this data set. The normal Probability plot for the device is plotted and shown in Figure 6.

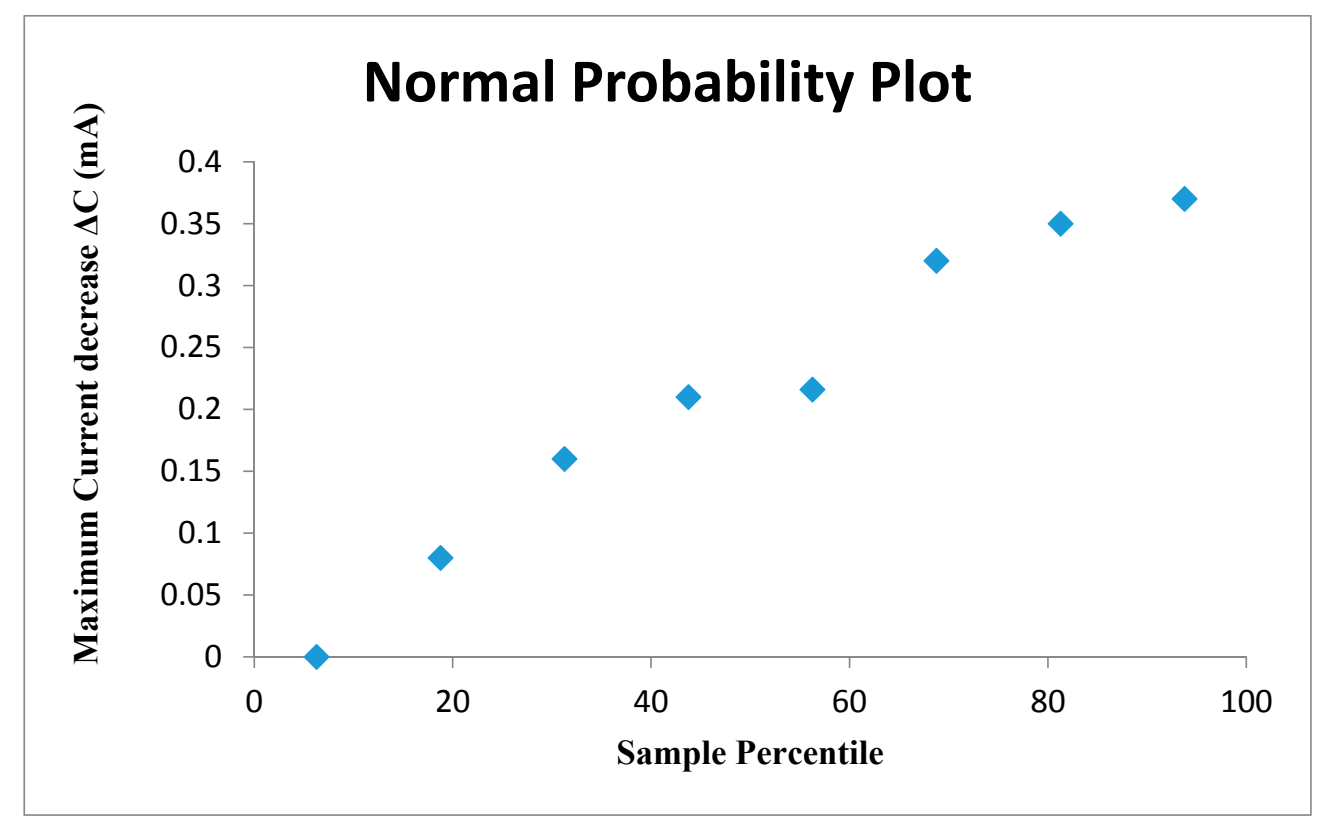

Fig. 6. Normal Probability Plot

On plotting a linear trend line between $\Delta \mathrm{C}$ and $\Delta \mathrm{O}_{2}$ the coefficient of determination $\mathrm{R}^{2}$ is found to be $99.57 \%$ with standard error of 0.081 shown in Figure 7 . The coefficient of determination $\mathrm{R}^{2}$ is useful because it gives the proportion of the variance (fluctuation) of one variable that is predictable from the other variable. It is a measure that allows us to determine how certain one can be in making predictions from a certain model. 
The coefficient of determination is a measure of how well the regression line represents the data. If the regression line passes exactly through every point on the scatter plot, it would be easy to explain all the variations.

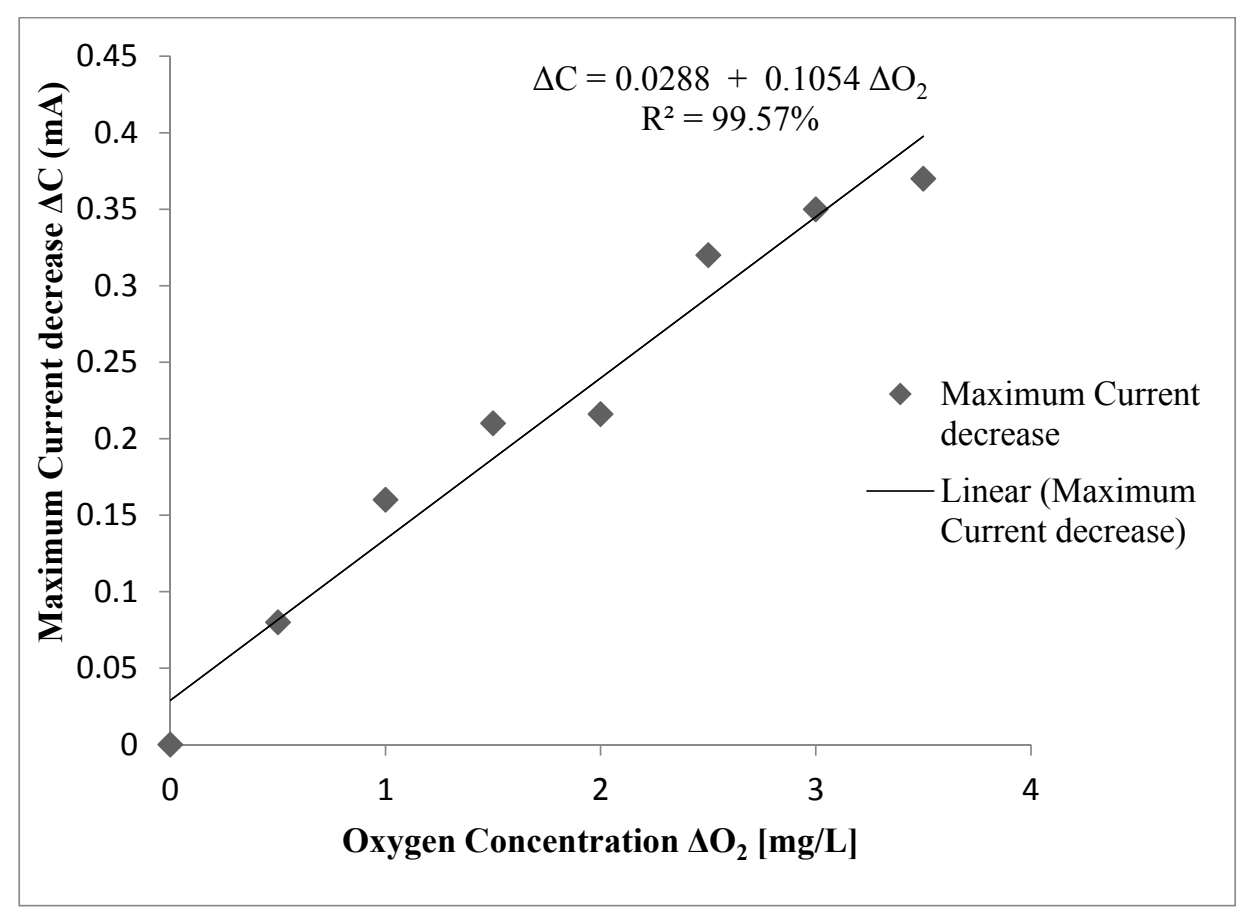

Fig.7. Trend line between $\Delta \mathrm{C}$ and $\Delta \mathrm{O}_{2}$ obtained from SPICE model

\section{Residual plot}

A residual plot between output and input shows that for a regression model to be good fit when residues are random. There should be no recognizable pattern. Good regression models give uncorrelated residuals.

The residual Plot for the device is plotted and shown in given Figure 8.

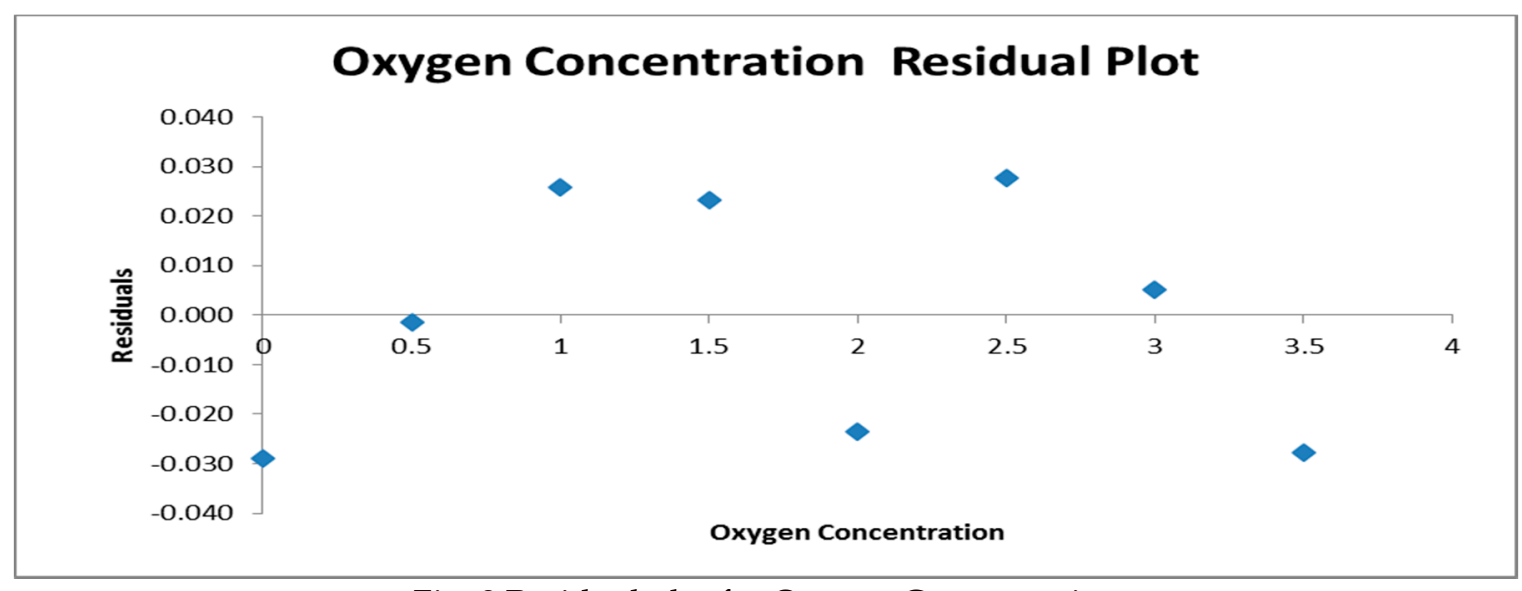

Fig. 8 Residual plot for Oxygen Concentration 


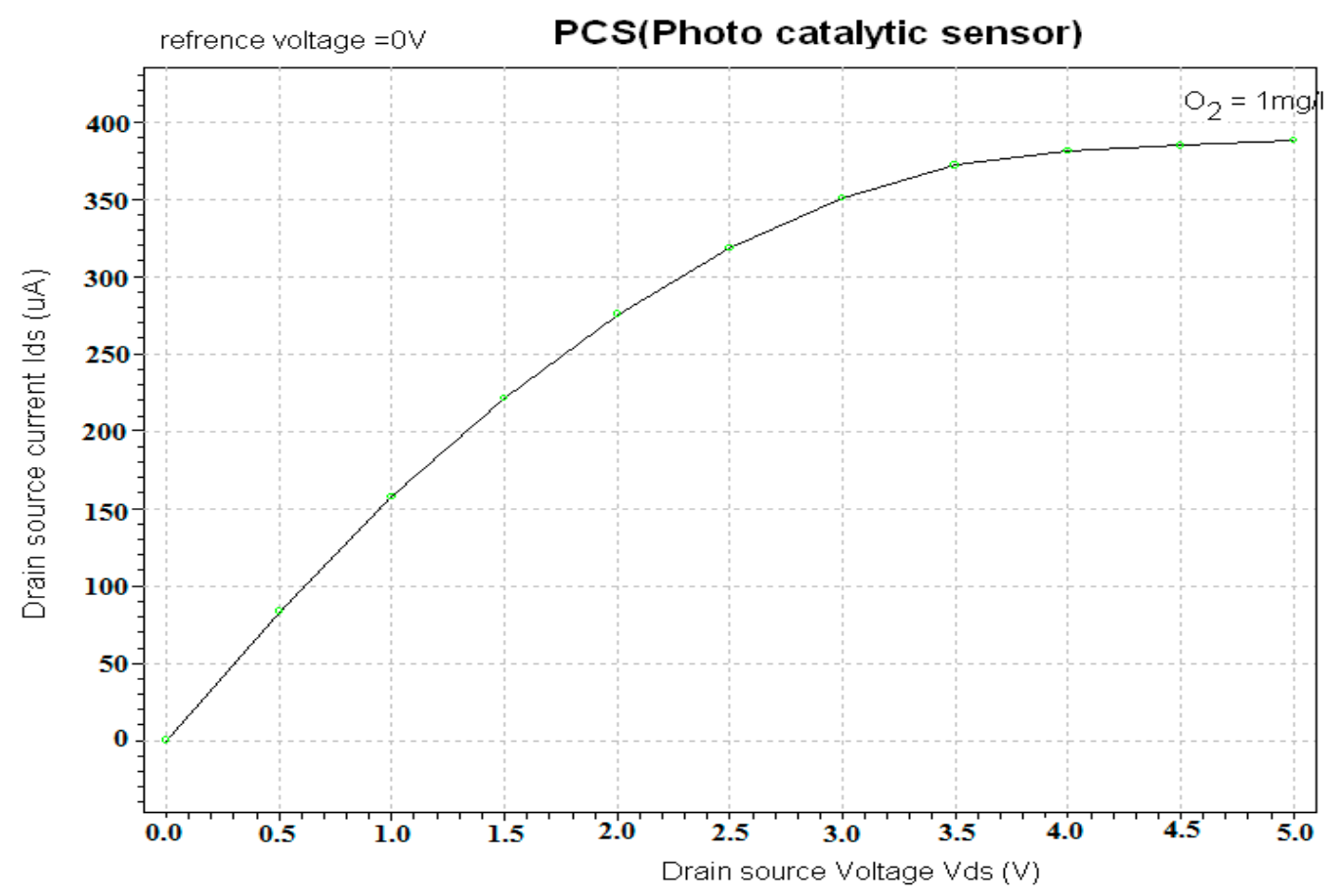

Fig. 9. Waveform between $\mathrm{Ids}_{\mathrm{ds}}$ and $\mathrm{V}_{\mathrm{ds}}$ at $\mathrm{O}_{2}=1 \mathrm{mg} / \mathrm{l}$

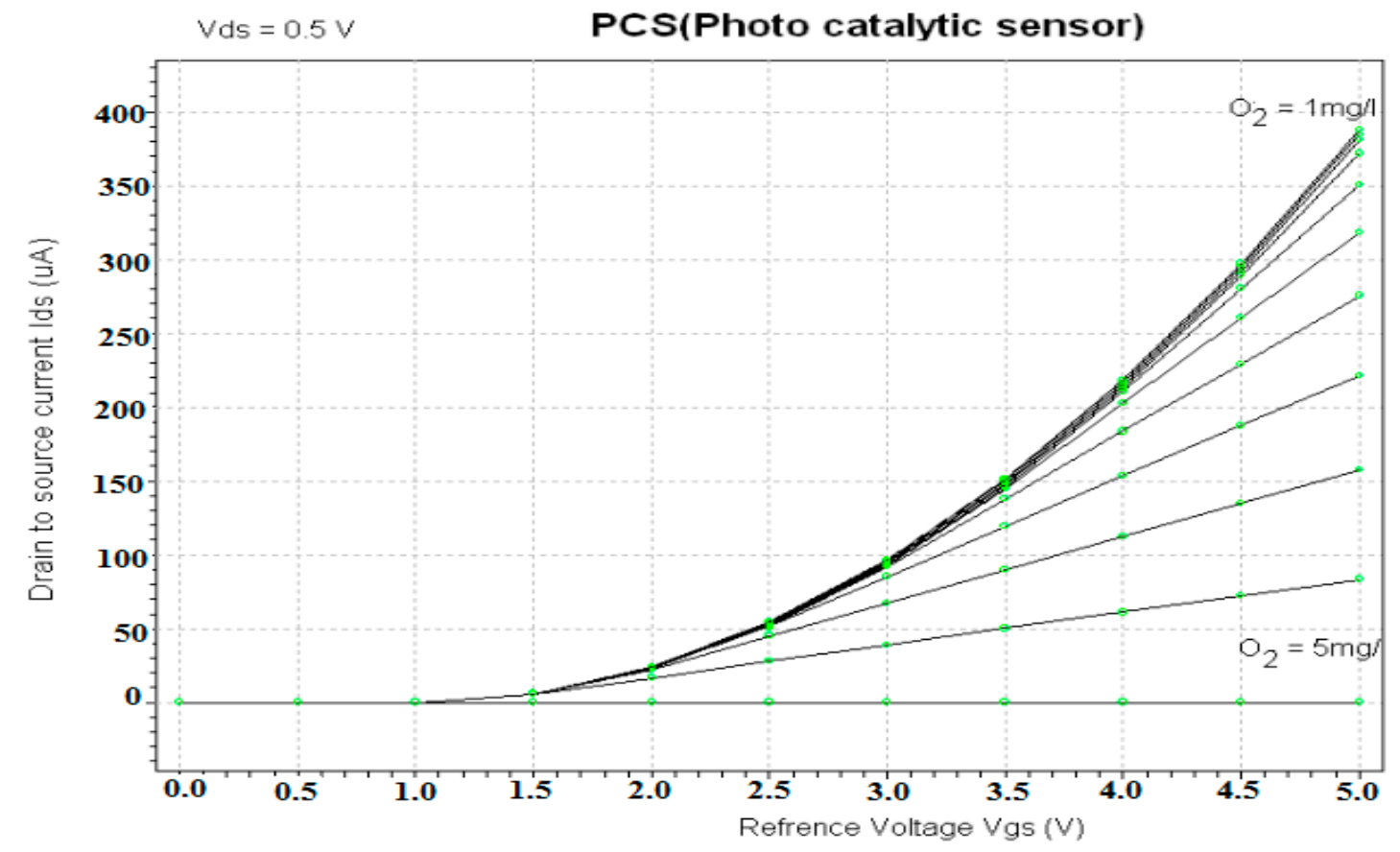

Fig.10. Waveform between $\mathrm{Id}$ and $\mathrm{Vgs}$ at $\mathrm{O}_{2}=1-5 \mathrm{mg} / \mathrm{l}$

\subsection{Results \& Discussion}

The simulation results have been compared both with experimental data and with the previously validated physicochemical model results. The relationship between drain current $\left(\mathrm{I}_{\mathrm{d}}\right)$ and gate to source voltage $\left(\mathrm{V}_{\mathrm{ds}}\right)$ is shown in Fig.9. in which at fixed value of $\mathrm{O}_{2}=1 \mathrm{mg} / 1$ the Value of $I_{d}$ varies directly with $V_{d s}$. also, the relationship of $I_{d}$ with $V_{g s}$ is shown in the Fig. 10 which shows that PCS exhibits linear characteristic 
in the range of $\mathrm{V}_{\mathrm{gs}}=2.5 \mathrm{~V}$ to $5.0 \mathrm{~V}$ this is an improvement over FIA. Fig.10. is a family of curves for $\mathrm{O}_{2}$ concentration from $1 \mathrm{mg} / 1$ to $5 \mathrm{mg} / \mathrm{l}$. The curves are drawn between $\mathrm{Ids}_{\mathrm{ds}}$ and $\mathrm{V}_{\mathrm{gs}}$ of PCS for $\mathrm{V}_{\mathrm{ds}}=0.5 \mathrm{~V}$ it can be seen that the proposed PCS is extremity linear for concentration more than $1 \mathrm{mg} / \mathrm{l}$ extending towards $5 \mathrm{mg} / \mathrm{l}$ of $\mathrm{O}_{2}$. This is an obvious advantage of this PCS model over the other models discussed in the introduction. The linear variation of Ids facilitates high accuracy measurements of quality of air. In addition the calibration of the instrument is also easier due to this linear behavior.

\section{Conclusion}

In this paper a simple and powerful approach to develop simulated computer models of bio- electronic sensors such as UVPCS using CMOS common source is shown. This approach relies on the Spice model .The developed macro model can be easily extended for simulating non- ideal behaviors and temperature dependence of the available sensors. This study may be extended and more improvement in terms of power and size can be achieved at layout level and thus more effective results may be obtained.

\section{References}

1. P. Whig and S. N. Ahmad, Simulation of linear dynamic macro model of photo catalytic sensor in SPICE, Compel the Int. J. Comput. Math. Electric. Electron. Eng. 33 (2014), 611-629.

2. P. Whig and S. N. Ahmad, Development of Economical ASIC For PCS For Water Quality Monitoring , JCSC Vol. 23, No. 6, 2014.

3. Sze, S.M.(1994), Semiconductor Sensors, Wiley, New York, NY.

4. P. Whig and S. N. Ahmad, "Performance analysis of various readout circuits for monitoring quality of water using analog integrated circuits," International Journal of Intelligent Systems and Applications, vol. 11, pp. 91-98, 2012.

5. Kim, Y.-C., Sasaki, S., Yano, K., Ikebukuro, K., Hashimoto, K. and Karube, I., “Photocatalytic sensor for the determination of chemical oxygen demand using flow injection analysis", Analytica Chimica Acta, Vol. 432 No. 2, pp. 59-66, 2001.

6. Duffy, J.A. (1990), Bonding Energy Levels and Bands in Inorganic Solids, Wiley, New York, NY.

7. Massobrio, G. and Antognetti, P. (1993), Semiconductor Device Modeling with SPICE, McGraw-Hill, New York, NY.

8. E. Rodriguez-Villegas, Low Power and Low Voltage Circuit Design with the FGMOS Transistor, vol. 20 of IET Circuits, Devices \& Systems Series, The Institution of Engineering and Technology, London, UK, 2006.

9. Peterson, M., Turner, J. and Nozik, A. , "Mechanistic studies of the photocatalytical behavior of $\mathrm{TiO} 2$ particles in photo electrochemical slurry and the relevance to photo detoxification reactions", Journal of Physical Chemistry B, Vol. 95, pp. 221-225,1991.

10. D. Kahng and S.M. Sze, "A oating-gate and its application to memory devices," The Bell System Technical Journal, vol. 46, no. 4, 1967, pp. 1288-1295.

11. Y. Berg, T. S. Lande, and S. Naess, "Low-voltage floating-gate current mirrors," in Proceedings of the 10th Annual IEEE International ASIC Conference and Exhibit, pp. 2124, September 1997 


\section{Appendix}

The SPICE model of a PCS includes a variety of parasitic circuit elements and some process related parameters in addition to the elements previously discussed in this paper. The syntax of a PCS includes several parameters, which can be specified to enhance the accuracy of the model. The commonly used parameter list is given below. The spice code used during the simulation process is also given below to specify the process and technology related parameters of the PCS. The SPICE parameters in this work have been set for the specific example. If some another sensitive layer of material is considered for PCS, then some electrochemical parameters have to be changed i.e., the dissociation constant, surface site densities and some other parameters values have to be adjusted.

\section{Spice code for PCS}

.model nmos external winfile="mos1.dll"

+ type=str("nmos")

$+\mathrm{Vto}=1.0 \quad \mathrm{Kp}=3.0 \mathrm{E}-5$

+ Phi $=0.65 \quad$ Lambda $=0.02$

$+\quad \mathrm{Nsub}=1.0 \mathrm{E}+15 \quad \mathrm{Nss}=1.0 \mathrm{E}+10$

Tox $=0.1 \mathrm{u}$

+ Tpg=1.00 Uo=700.0

$\mathrm{Ld}=0.8 \mathrm{u}$

$+\mathrm{Kf}=1.0 \mathrm{E}-26$

Is $=1.0 \mathrm{E}-15$

Af $=1.2$

$+\mathrm{Pb}=0.75$

$\mathrm{Cj}=2.0 \mathrm{E}-4$

Js $=1.0 \mathrm{E}-8$

$+\quad$ Cjsw $=1.00 \mathrm{E}-9$

$\mathrm{Mjsw}=0.33$

$\mathrm{Mj}=0.5$

$+\mathrm{Cgbo}=2.0 \mathrm{E}-10$

Cgdo $=4.00 \mathrm{E}-11$

$\mathrm{Fc}=0.5$

$+\mathrm{Rd}=10.0$

$\mathrm{Rs}=10.0$

Cgso=4.00E-11

$\mathrm{Rsh}=30.0$

\section{.SUBCKT PCS 6134101}

*drain $<$ ref.el<source $<$ bulk $<$ PCS

$+\mathrm{q}=1.6 \mathrm{e}-19 \mathrm{NAv}={ }^{\prime} 6.023 \mathrm{e} 23^{*} 1 \mathrm{e} 3^{\prime}$

+ epsw $=78.5$

+ Cbulk $=0.1$ Cox $=0.2 \quad \mathrm{Cq}=0.3$

+ Eabs=4.7 Phim=4.7 Erel=0.200 Chieo=3e-3 Philj=1e-3

$+\mathrm{ET}=\mathrm{\prime} /\left(\mathrm{k}^{*} \mathrm{~T}\right)^{\prime}$

+ sq $=$ 'sqrt $\left(8^{*} \text { eps } 0^{*} \text { epsw }^{*} \mathrm{k}^{*} \mathrm{~T}\right)^{\prime}$

$+\mathrm{KK}={ }^{\prime} \mathrm{Ka}^{*} \mathrm{~Kb}^{\prime}$

$+\mathrm{C}_{\mathrm{M}}=1 /(1 / \mathrm{Cox}+1 / \mathrm{Cq})^{\prime}$

$+\mathrm{Cin}=\mathrm{Cgs}+\mathrm{CM} 1$

+ Cout $=\mathrm{Cds}+\mathrm{CM} 2$

Eref 110 VOL='Eabs-Phim-Erel+Chieo+Philj'

Ceq $102 \mathrm{C}=' 1 /(1 / \mathrm{Cox}+1 / \mathrm{Cq})^{\prime}$

.end

$\mathrm{x} 1 \mathrm{~d} \mathrm{~g} \mathrm{~s} \mathrm{~b} \mathrm{nmos} \mathrm{l}=5 \mathrm{u} \mathrm{w}=5 \mathrm{u}$

$\operatorname{vdd} 05$

$\operatorname{vg} g 05$

vs $\mathrm{s} 00$ 
$v b$ b 00

.de vd 050.1 vg 050.5

.print dc i1(x1)

.ends

(C) 2016 by the authors; licensee Preprints, Basel, Switzerland. This article is an open access article distributed under the terms and conditions of the Creative Commons by

Attribution (CC-BY) license (http://creativecommons.org/licenses/by/4.0/). 